\title{
La documentation en milieu communautaire au Québec : entre menaces et avancées
}

Community Based Documentation Centres in Quebec: Between Threats and Opportunities

\section{La documentación en el medio comunitario en Québec: entre amenazas y oportunidades}

Julie Leclair

Volume 54, numéro 2, avril-juin 2008

Topographie du Québec documentaire

URI : https://id.erudit.org/iderudit/1029327ar

DOI : https://doi.org/10.7202/1029327ar

Aller au sommaire du numéro

Éditeur(s)

Association pour l'avancement des sciences et des techniques de la documentation (ASTED)

ISSN

0315-2340 (imprimé)

2291-8949 (numérique)

Découvrir la revue

Citer cet article

Leclair, J. (2008). La documentation en milieu communautaire au Québec : entre menaces et avancées. Documentation et bibliothèques, 54(2), 159-163. https://doi.org/10.7202/1029327ar
Résumé de l'article

Après plus de 40 ans de pratiques communautaires et à l'ère des partenariats et de la médiation, le portrait des centres de documentation en milieu communautaire n'est pourtant guère reluisant. Quelles sont les particularités de la documentation en milieu communautaire ? Quels rôles jouent les centres de documentation présents dans ce milieu et quels sont les enjeux auxquels ils doivent faire face?
Tous droits réservés (C) Association pour l'avancement des sciences et des techniques de la documentation (ASTED) et Corporation des bibliothécaires professionnels du Québec (CBPQ), 2008
Ce document est protégé par la loi sur le droit d'auteur. L'utilisation des services d’Érudit (y compris la reproduction) est assujettie à sa politique d'utilisation que vous pouvez consulter en ligne.

https://apropos.erudit.org/fr/usagers/politique-dutilisation/ 


\title{
La documentation en milieu communautaire au Québec : entre menaces et avancées
}

\author{
JULIE LECLAIR
}

Responsable du développement des collections

Centre de documentation sur l'éducátion des adultes et la condition féminine (CDÉACF)

leclair@cdeacf.ca

\begin{abstract}
RÉSUMÉ | ABSTRACTS | RESUMEN
Après plus de 40 ans de pratiques communautaires et à l'ère des
partenariats et de la médiation, le portrait des centres de docu-
mentation en milieu communautaire n'est pourtant guère relui-
sant. Quelles sont les particularités de la documentation en milieu
communautaire? Quels rôles jouent les centres de documenta-
tion présents dans ce milieu et quels sont les enjeux auxquels ils
doivent faire face?
Community Based Documentation Centres in Quebec: Between Threats and Opportunities

Following 40 years of community practices in an era of partnerships and mediation, the portrait of community-based documentation centres is far from brilliant. What are the particularities of community based information resource centres? What roles do documentation centres in these milieus play and what issues must they face?

La documentación en el medio comunitario en Québec: entre amenazas $y$ oportunidades

Después de más de 40 años de prácticas comunitarias y en la era de la colaboración y de la mediación, el retrato de los centros de documentación en el medio comunitario no es de lo más reluciente que digamos. ¿Cuáles son las particularidades de la documentación en el medio comunitario ? ¿Qué rol desempeñan los centros de documentación presentes en este medio y cuáles son los retos que deben enfrentar?
\end{abstract}

$\mathrm{P}$ OUR COMPRENDRE LES PARTICUlarités de la documentation en milieu communautaire, il faut d'abord définir ce dernier.

La naissance du mouvement d'action communautaire remonte aux années 1960, au moment où la modernité confronte radicalement le Québec. Des comités de citoyens s'organisent alors pour solutionner des problèmes quotidiens circonscrits tels que la salubrité des immeubles, la circulation automobile et l'endettement. Petit à petit, ces groupes populaires, appelés aujourd'hui organismes communautaires, étendent leurs champs d'intérêt et visent des problèmes sociaux plus vastes, comme la violence envers les femmes et les questions environnementales.

Présents dans une multitude de secteurs dont la santé, l'emploi et l'éducation, ces organismes expérimentent des pratiques alternatives à celles généralement mises en œuvre par le réseau institutionnel - écoles, hôpitaux, etc. 一, tout en demeurant en marge des organisations politiques et syndicales.

Comme leur nom l'indique, les organismes communautaires, sans but lucratif, doivent impérativement être enracinés dans la communauté. Ils entretiennent une vie associative et démocratique et sont libres de déterminer leur mission, leurs pratiques et leurs orientations. Leurs actions sont guidées par la prise en charge citoyenne des problèmes sociaux afin d'améliorer les services collectifs et d'obtenir de meilleures conditions de vie.

Le milieu communautaire québécois comprend aujourd'hui 8 ooo organismes actifs tant au niveau local que régional ou national. La majorité de ces organismes font partie de divers regroupements à la fois sectoriels (reliés directement à leur secteur d'intervention, par exemple la santé) et intersectoriels (regroupement de plusieurs organismes rattachés à divers secteurs d'intervention, mais associés à une même région).

\section{La documentation en milieu communautaire}

Pour réaliser leur travail tant d'analyse que de représentation ou de formation, les 50 ooo personnes employées dans le milieu communautaire ont besoin d'avoir accès rapidement à des faits concrets, documentés et chiffrés, bref, à de la documentation à jour. 


\section{La naissance du mouvement d'action communautaire remonte aux années 60 , au moment où la modernité frappe le Québec de plein fouet. 00000000000000000000000000000000000000000}

"Connaître les plus récentes recherches réalisées dans le domaine de la violence faite aux femmes est essentiel pour rediffuser de l'information auprès de nos membres $»^{1}$, affirme Caroline Farly, agente de liaison et de promotion à la Fédération des ressources d'hébergement pour femmes violentées et en difficulté du Québec.

Non seulement le milieu communautaire a besoin de consulter des documents, mais il en crée également. En effet, la mission des organismes communautaires les amène régulièrement à produire une panoplie de documents : bulletins et revues, mémoires pour une commission parlementaire, documents de réflexion portant sur un enjeu de société, guides de formation, recherchesaction, recueils de témoignages, etc.

Tous ces documents doivent nécessairement être regroupés quelque part afin d'éviter que ce patrimoine ne soit éparpillé et, d'une certaine manière, inaccessible.

"Il nous est difficile de trouver des documents portant sur le secteur de l'emploi dans le communautaire. Pourtant, nous savons que les organismes en produisent, mais ils se ramassent souvent sur une tablette obscure. C'est une perte pour la mémoire collective ", déplore Martin Frappier, de la Coalition des organismes communautaires pour le développement de la main-d'œuvre.

\section{Portrait des centres de documentation en milieu communautaire}

Pour pallier ce problème, quelques organismes ont mis sur pied des centres de documentation ainsi qu'une panoplie de services de documentation appelés tablettes, bibliothèques maison, coins de lecture, bibliothèques roulantes, etc. En réalité, «il y a peu de centres de documentation, mais il y a beaucoup de documents", résume Bernard Vallée, fondateur du Centre populaire de documentation de Montréal et aujourd'hui responsable des activités pédagogiques à L'Autre Montréal.

Il est difficile de tracer un portrait général des centres de documentation en milieu communautaire puisqu'il n'existe ni statistiques ni répertoire à leur sujet. Avant tout, ces centres sont des bibliothèques spécialisées dans un domaine précis mais dont le mandat n'est

1. Les citations dans le texte sont, sauf indication contraire, tirées d'entrevues réalisées par l'auteure. pas forcément d'acquérir des documents produits par le milieu communautaire. Le centre de documentation de l'Institut québécois sur la déficience intellectuelle en serait un exemple, tout comme celui de la Fédération québécoise de l'autisme. Ceux-ci comblent un manque en conservant des documents rares, en français, souvent issus de la "littérature grise " et présentant des points de vue alternatifs sur leur champ d'expertise que l'on ne trouve pas nécessairement dans les bibliothèques publiques ou dans les bibliothèques universitaires. Ils disposent généralement d'un budget d'acquisition plutôt faible, voire inexistant dans certains cas. Ils reçoivent toutefois beaucoup de documents gratuits et d'autres documents sous forme de dons.

Plusieurs organismes communautaires, dont L'Autre Montréal, le Front d'action populaire en réaménagement urbain ou le Regroupement des cuisines collectives, disposent d'un centre de documentation pour répondre à leurs propres besoins internes et ne sont pas ouverts au public. Les centres de documentation accessibles au public, comme le Centre de documentation sur l'éducation des adultes et la condition féminine ( $\mathrm{CDÉACF})$, desservent quant à eux plusieurs types de clientèle : intervenants communautaires, étudiants, chercheurs, parents, enseignants, professionnels de la santé, journalistes, fonctionnaires, etc. Bref, la clientèle n'est pas nécessairement issue du milieu communautaire.

Les centres de documentation visités dans le cadre de l'élaboration de cet article sont en général assez peu fréquentés, du moins sur place. Par exemple, le centre de documentation de la Fédération des centres d'action bénévole du Québec, qui dispose d'une collection d'environ 20000 documents, reçoit une trentaine de personnes par année, selon son directeur général Pierre Riley.

La taille des fonds documentaires varie considérablement, allant de quelques dizaines de titres à plusieurs dizaines de milliers. Ils sont plus ou moins organisés selon les normes bibliothéconomiques reconnues. Les centres adoptent souvent un système maison de classification et d'indexation. Quant aux règles de catalogage, elles demeurent très peu appliquées. Aucun des organismes contactés dans le cadre de cet aírticle, à l'exception du CDÉACF, n'a d'ailleurs élaboré de politique de développement des collections par écrit. Plusieurs personnes interrogées ont signalé des difficultés à mettre à jour leur catalogue, rarement accessible sur Internet. On comprend alors pourquoi certaines éprouvent de la difficulté à quantifier le nombre de documents dont elles disposent...

$\mathrm{Ce}$ constat n'est guère étonnant compte tenu des circonstances. Les travailleurs du milieu communautaire ont beau être flexibles, polyvalents et bien connaitre leur champ d'expertise, ils doivent néanmoins composer avec un budget famélique et fonctionner en petites équipes de trois ou quatre personnes. Il y a en outre peu de personnel formé en bibliothéconomie 
ou en documentation, et la responsabilité d'assurer le fonctionnement du' centre de documentation n'est pas toujours rattachée clairement à une personne précise.

\section{L'enjeu principal : mieux diffuser les savoirs}

Les organismes communautaires produisent beaucoup de documents, là n'est pas le problème. Aujourd'hui encore, ils omettent trop souvent de diffuser leur production. Pourquoi ? D'entrée de jeu, beaucoup d'organismes ignorent tout simplement que leurs publications sont assujetties à la loi sur le dépôt légal. Parfois, la mauvaise perception qu'ils ont de leurs propres documents, souvent fondée sur des critères esthétiques (mise en page qui n'est pas à la fine pointe sur le plan graphique, par exemple), fait en sorte qu'ils jugent inutile de les diffuser, à tort il va sans dire.

Pour Bernard Vallée, responsable des activités pédagogiques à L'Autre Montréal et fondateur du Centre de documentation populaire (CDP) de Montréal, le problème va plus loin. Il est surtout lié à un manque de vision globale. À l'ère de la mondialisation, le milieu communautaire québécois souffre encore de courte vue. À son avis, le localisme est l'un des pires obstacles à la diffusion des documents produits par le milieu communautaire. "Malheureusement, affirme-t-il, le localisme se fonde sur toutes sortes de raisons, notamment sur la concurrence par rapport au financement qui est insuffisant, alors que nous pourrions nous inspirer de ce que les autres ont fait avant nous. Il faudrait reprendre le slogan environnemental Penser globalement, agir localement. »

Plusieurs personnes interrogées ont également souligné l'importance de sensibiliser et de mobiliser les acteurs du milieu communautaire sur la reconnaissance de leurs savoirs et de leur savoir-faire. "J'espère que le milieu communautaire prendra conscience qu'il ne transforme pas seulement son petit patelin, mais qu'il a aussi un pouvoir de transformation mondiale ", souhaite Rosalie Ndejuru, directrice générale du CDÉACF. « C'est un travail de longue haleine. Il faut des associations, comme la Fédération des centres d'action bénévole du Québec, pour sensibiliser et créer des outils qui vont servir d'exemples », estime de son côté Pierre Riley.

Pour collecter et diffuser la production documentaire et les savoirs de manière dynamique, il faut donc se munir d'une interface efficace. Les bibliothèques publiques ne peuvent à elles seules répondre à ces besoins. Elles n'ont pas le mandat d'acquérir les productions documentaires des organismes communautaires, ni l'espace physique suffisant.

Quant à Internet, il constitue plus un outil qu'une finalité en soi, et ce, même si quelques travailleurs en milieu communautaire considèrent Internet comme le centre de documentation par excellence. Il est vrai que la plupart des publications gouvernementales, source

\section{Il est difficile de tracer un portrait général des centres de documentation en milieu communautaire puisqu'il n'existe ni statistiques ni répertoire à leur sujet.}

majeure d'information pour le milieu communautaire, sont maintenant disponibles sur Internet. Or, pour Pierre Riley, «il y a effectivement plein d'information sur Internet, mais c'est artificiel, car cela peut disparaître du jour au lendemain sans laisser de traces ». Pour Anne St-Cerny, coordonnatrice à Relais-femmes et qui travaille dans le milieu communautaire depuis 25 ans, les centres de documentation demeurent toujours à cet égard irremplaçables : "Beaucoup de gens n'ont pas accès à Internet. C'est un outil qui n'offre pas de tri de l'information, pas de guide, pas de contact humain. Tandis qu'un centre de documentation a le mandat de sélectionner, de trier et d'organiser. »

Le flou entourant la perception par. le milieu communautaire de l'importance de ses centres de documentation met en péril leur bon fonctionnement, voire leur existence, et du coup nuit à la diffusion des savoirs. L'un des principaux enjeux auxquels fait face la documentation en milieu communautaire concerne justement la pérennité des centres de documentation. Ces derniers souffrent de problèmes criants de visibilité et de financement. Afin d'assurer leur survie, ils doivent d'abord rappeler leur importance non seulement aux yeux des principaux bailleurs de fonds, mais à ceux du milieu communautaire lui-même. "Les bibliothèques et centres de documentation sont essentiels, d'une part, pour constituer notre mémoire collective et faire la promotion des savoirs communautaires, surtout dans le contexte du développement des sociétés dites de savoir. Ce n'est pas seulement l'institution universitaire qui détient le savoir. D'autre part, c'est un enjeu citoyen politique de maintenir des centres de documentation, qui ne sont pas juste là pour archiver, mais qui animent la production et la diffusion de connaissances et de savoirs critiques ", estime Sylvie Jochems, professeure à l'École de travail social de l'UQAM.

\section{L'argent, le nerf de la guerre}

Un catalogue sur fiches, un budget d'acquisition annuel de 1 ooo\$, quelques rares personnes qui se présentent sur place : voilà la réalité à laquelle est confronté Réal Bathalon, coordonnateur des services de documentation et de référence à l'Institut interculturel de Montréal depuis 1977. "Ce n'est vraiment pas rose, nous n'avons pas beaucoup de ressources matérielles, humaines et informatiques pour travailler ", déploret-il. Malgré des ressources limitées, la bibliothèque 
L'un des principaux enjeux auxquels fait face la documentation en milieu communautaire concerne justement la pérennité des centres de documentation. Ces derniers souffrent de problèmes criants de visibilité et de financement.

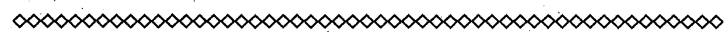

offre un service de référence prisé et répond à plusieurs centaines de demandes par année.

On ne s'étonnera pas que la faible reconnaissance de l'importance du rôle des centres de documentation, tant par les différents paliers gouvernementaux que par les autres bailleurs de fonds, entraîne de lourdes conséquences sur leur fonctionnement. Les difficultés de financement des centres de documentation en milieu communautaire ne datent pas d'hier. Le Centre populaire de documentation de Montréal - pour ne citer qu'un exemple - a dû fermer ses portes à la fin des années 1980, faute de financement adéquat. Fondé par des groupes populaires en 1976, il avait pour objectif de réunir des documents témoins des activités des groupes populaires et de les rendre accessibles auprès de ses membres et du public.

Avec l'adoption de la politique sur l'action communautaire en 2001, le gouvernement québécoís démontre sa volonté de mieux soutenir la mission des organismes communautaires. Mais le Secrétariat à l'action communautaire autonome et aux initiatives sociales, appareil gouvernemental chargé d'appliquer cette politique, n'offre aucun programme de financement spécifique destiné au fonctionnement des centres de documentation en milieu communautaire.

Pour assurer la survie de leur centre de documentation, les organismes doivent donc multiplier les demandes de subvention tout en diversifiant leurs sources de revenus, notamment grâce au membership et à l'autofinancement. Par exemple, le centre de documentation de l'Institut québécois de la déficience intellectuelle utilise un mode de financement basé sur la perception des cotisations annuelles versées par ses membres. De son côté, la Fédération des centres d'action bénévole du Québec dispose d'une librairie en ligne de livres spécialisés en action communautaire dont les profits s'élèvent à environ 3000 \$ par année.

Or, un meilleur financement pourrait aider quelqu'un comme Réal Bathalon à embaucher plus d'employés, à les former adéquatement, à mettre son catalogue en ligne, etc. Bref, tout le milieu communautaire gagnerait à mieux reconnaître l'importance des centres de documentation dans la diffusion de savoirs spécialisés et alternatifs, ce qui attirerait sans doute par le fait même une attention accrue des principaux bailleurs de fonds. Voilà un enjeu majeur auquel fait face la documentation en milieu communautaire.
Accompagner les organismes communautaires dans la diffusion de leurs savoirs est fondamental, que ce soit par le biais de sessions de formation portant sur l'édition d'un document ou sur le développement d'un centre de documentation. À l'heure des départs massifs à la retraite des baby boomers et des enjeux liés au transfert des savoirs et au moment où le milieu communautaire québécois se professionnalise de plus en plus, les défis de la documentation en milieu communautaire sont donc nombreux.

\section{Que peuvent offrir les bibliothèques publiques aux organismes communautaires?}

«Les bibliothèques sont de plus en plus actives hors les murs et se tournent de plus en plus vers les clienteles non traditionnelles. Pour aller chercher les gens là où ils sont, il faudrait des bibliothécaires qui iraient rencontrer les organismes, qui connaissent le milieu et la culture du communautaire ", fait valoir Dominique Malchelosse, agente pour Alpha-biblio, un projet amorcé en 2006 qui vise entre autres à créer des liens entre les bibliothèques publiques et les organismes communautaires d'alphabétisation. Pour attirer la clientèle des organismes communautaires, quelques initiatives ont aussi vu le jour : par exemple, la bibliothèque de l'Assomption offre des services documentaires adaptés aux besoins du Regroupement des aidants naturels du comté de L'Assomption, et la bibliothèque de Sorel-Tracy délègue des membres de son personnel dans l'organisme Carrefour jeunesse-famille pour sensibiliser les parents et éveiller les enfants à l'écrit.

\section{Un cas d'exception : le CDÉACF}

Fondé en 1983, ce centre de documentation a comme particularité de ne relever d'aucun organisme et d'avoir dûment inscrit dans son énoncé de mission la collecte des documents issus du milieu communautaire qui concernent ses champs d'expertise (éducation et alphabétisation des adultes, condition féminine et action communautaire). Ses collections se sont enrichies considérablement au fil des ans grâce au dépôt d'autres collections provenant de plusieurs regroupements et fédérations d'organismes communautaires. Il emploie des bibliothécaires professionnelles et des techniciennes en documentation afin de gérer ses 100000 documents. L'arrivée des technologies de l'information et de la communication à la fin des années 1990 lui a permis de développer plusieurs portails thématiques ainsi qu'une bibliothèque virtuelle consacrée à la diffusion sur Internet de documents produits par le milieu communautaire.

L'auteure désire remercier les personnes des organismes suivants qui ont fourni des informations utiles à la rédaction de l'article. 
- L'Autre Montréal, Bernard Vallée

- Bibliothèque et Archives nationales du Québec, Mireille Laforce

- Bibliothèque ville de l'Assomption, Eve Lagacé

- Bibliothèque ville de Sainte-Thérèse, Lise

Thériault et Nicole Bouchard

- Bibliothèque ville de Sorel-Tracy, Guy

Desjardins

- Bibliothèques de l'arrondissement de MontréalNord, Cécile Lointier

- Centre de documentation sur l'éducation des adultes et la condition féminine, Rosalie Ndejuru

- Coalition des organismes communautaires pour le développement de la main-d'œuvre, Martin Frappier

- École de travail social de l'UQAM, Sylvie Jochems

- Fédération des centres d'action bénévole du Québec, Pierre Riley et Patricia Morin

- Fédération des ressources d'hébergement pour femmes violentées et en difficulté du Québec, Caroline Farly

- Fédération québécoise de l'autisme, Geneviève Gagnon

- Front d'action populaire en réaménagement urbain, François Saillant
- Institut interculturel de Montréal, Réal Bathalon

- Institut québécois de la déficience intellectuelle; Claude F. Leclair

- Projet alpha-biblio, CDÉACF, Dominique Malchelosse

- Regroupement des cuisines collectives, Isabelle Phaneuf

- Relais-femmes, Anne St-Cerny

- Le Traversier, Chantal Pelletier

- Union des consommateurs, Brigitte Alexandre Bussières $(-)$

\section{Sources consultées}

Bourque, Denis. 2007. L'organisation communautaire: fondements, approches et champs de pratique. Québec: Presses de l'Universifé du Québec, 534 p.

Caron, Joseph. 1997. Les besoins d'information dans les groupes communautaires lorsque le besoin en matière d'information se confond avec sa production. Documentation et bibliothèques, oct.-déc. $1997: 169$-178.

Duval, Michelle [et al.]. 2005. Les organismes communautaires au Québec : pratiques et enjeux. Montréal : Gaëtan Morin Éditeur, $164 \mathrm{p}$.

Secrétariat à l'action communautaire autonome et aux initiatives sociales http://www.mess.gouv.qc.ca/saca/ (consultée sur Internet le 2 décembre 2007).

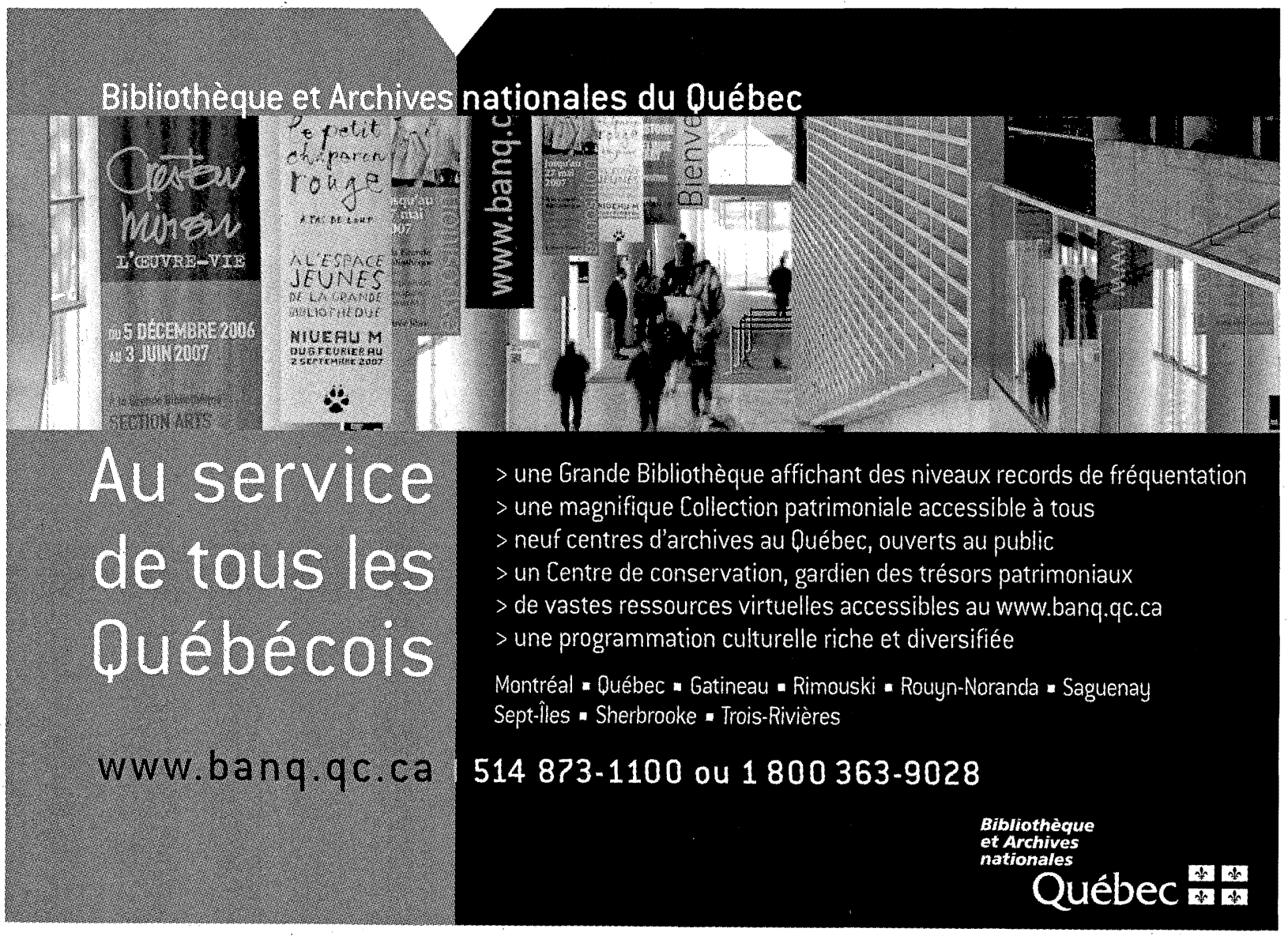

